\title{
Effective use Method of Cloudlet Resources by Mobile Users
}

\author{
Rashid G. Alakbarov \\ Institute of Information Technology of ANAS /Department, Baku, AZ1141, Azerbaijan \\ E-mail: rashid@iit.ab.az \\ Oqtay R. Alakbarov \\ Institute of Information Technology of ANAS /Department, Baku, AZ1141, Azerbaijan \\ E-mail: oqtayalakbarov@yahoo.com
}

Received: 07 October 2017; Accepted: 08 January 2018; Published: 08 February 2018

\begin{abstract}
The paper explores the architecture of mobile cloud technologies. The advantages and the problems emerging during the use of this technology are analyzed. The study analyzes the establishment of network infrastructure based on cloudlets at the second level of mobile computation clouds with hierarchic structure. At the same time, the issues of computation and meeting the demand for memory resources of mobile devices are investigated by employing this technology. The paper presents the solution of the linking problem of cloudlets located in the proximity of base stations of wireless metropolitan area networks (WMAN). The study also determines the number of communication channels among cloudlets for the purpose of effective utilization of cloudlet resources. The issue of uploading necessary program extensions to a corresponding cloudlet by using the possible number specifying the importance of cloudlets was investigated and a method was proposed.
\end{abstract}

Index Terms-Mobile computation clouds, mobile devices, computation and memory resources, cloudlet, computation clouds, communication channel, cloud services.

\section{INTRODUCTION}

At present, comprehensive research is being carried out on the efficient use of computing and memory resources of data processing centers by the means of Cloud Computing technologies in the world. Such systems executing large computations and possessing memory resources are created on the basis of computer networks with high-speed communication channels. Cloud Computing technology enables the more efficient use of computing and memory resources. User information is stored in servers of computing clouds, processed and the results is provided via browsers [1,2]. Cloud Computing service enables the widespread use of clustering and virtualization of computing and memory resources of processing. Recently, mobile users have started to use the services of cloud computing technologies broadly.
The paper studies the issues of more efficient use of resources of computing clouds by employing mobile Cloud Computing technologies widely used in recent period. The rapid expansion of the use of mobile devices (laptops, tablets, smartphones etc.) and connection to the internet via corresponding telecommunication technologies (GPS, 3G/4G, Wi-Fi, etc.) has given an impetus to the development of mobile Cloud Computing technology. It is known that, the capabilities of any mobile device (computation and memory resources) are limited. However, users apply these devices during the solution of problems requiring large computing and memory resources. For this purpose, cloud computing technologies are used broadly. Hence, limitations of computing and memory resources in mobile user devices can be eliminated by employing cloud technologies.

\section{ARChitecture of Mobile Cloud Technologies}

Mobile cloud computing is a new platform emerged as a result of integration of mobile devices and computing clouds and enables the users to solve complex problems and store large-volume data in the memory in clouds [3].

Technical capabilities of mobile devices (computing and memory resources) are limited. On one hand, users require high computing resources and at the same time, desire to use small-size mobile devices. Cloud technologies are broadly used in order to eliminate this limitation. Mobile device users can carry out the solution of a problem by using the facilities of cloud technologies. The availability of cloud servers at cheaper prices in recent times facilitates the broad use of such services by mobile users. At present, several companies develop many extensions for mobile device users (Google, Gmail, Maps and Navigation systems for Mobile, Voice Search, Mobil Me from Apple, Live Mesh from Microsoft), which enables the broad use of mobile cloud computing by users [4].

The processing and storage of information in mobile computing clouds is carried out outside mobile devices. Mobile devices in mobile computing clouds are connected to computing clouds system via internet 
network with the help of base stations (GPS, 3G/4G, Wi$\mathrm{Fi}$, etc.) and use the required services. Currently, users use three types (IaaS, PaaS and SaaS services) of cloud services widely. IaaS (Infrastructure as a Service) enables the users to use computing and memory resources of cloud systems (for example: Amazon Elastic Cloud Computing - EC2 and Amazon S3 - Simple Storage Service). PaaS (Platform as a Service) is a platform facilitating the use of operating systems located in virtual servers and specialized software extensions (Google App Engine, Microsoft Azure, My SQL, etc.) by users. SaaS (Software as a Service) users can solve their problems by using software tools (Google Apps, Google Docs, Autodesk, etc.) located in servers of computing clouds. SaaS user can obtain the resident part of required software tool without downloading that software tool and launching it in servers of computing clouds via internet network. Software extensions operate in servers of providers maintaining SaaS service and send the processed information to user. Hence, user does not purchase software tool and is charged for the use. Currently, millions of mobile users broadly use mobile extensions (mobile commerce, mobile education, mobile health, mobile games, etc.) by using the services of mobile cloud providers [3,5]. Developed mobile extensions do not depend on operating systems of mobile devices and the type of device. Hence, the number of mobile users using the services of cloud technologies increases rapidly day after day. The primary goal of mobile computing clouds is to facilitate the users to use computing clouds conveniently and rapidly. In this case, mobile devices possessing minimal computing and memory resources act as a client terminal connected to Internet network.

According to estimations of analysts of Gartner company, the number of internet users using mobile devices will reach 2,16 billion users in 2016. This figure will rise up to 2,56 billion users in 2018. This will facilitate the rapid increase of the number of mobile internet users.

At present, centralized (traditional) clouds used by users are not capable of delivering the processed information to users rapidly notwithstanding the large computing and memory resources of those. The rapid increase in number of mobile users in computing clouds leads to the overload of network which causes delays in delivering the processed information to users. In order to eliminate the above mentioned drawback, the resources of computing clouds must be located in close proximity to users [6].

While using the services of computing clouds, a user can be in two regimes: offline and online. Assume that, a user obtains virtual computing machine from cloud for problems requiring large computing capacity. Cloud sends the problems and obtains the result after a while. In this regime, there is no direct connection between computer and the cloud necessary for the solution of the problem till the required time. However, there are some problems, for which the connection is present until the process is finalized between the user and the cloud (in online regime). Hence, the solution of such problems leads to the overload of network. In order to eliminate the network overloads, mobile computing clouds with hierarchic structure are used. The architecture of mobile computing clouds with hierarchic structure is given in figure 1. As seen, the architecture of mobile computing clouds consists of several components: mobile users (Mobil Device, Smartphone, etc.), mobile communication operators (Cellular Networks), wireless connection devices - Access Point (Wi-Fi,3G/4G), cloudlet, computing cloud providers (Amazon, İBM, Google, Microsoft, etc.) [7]. As seen in the scheme, mobile users are connected to Cloud Computing system via cloudlets located near base stations (Cellular Networks, Wi-Fi- Access Point) and employ its computing and memory resources. Cloudlet (small computing clouds) is a device (server) located in close proximity to users and provides the more rapid loading and solution of user information from central servers. After the user has completed his/her work, information is uploaded to server again. Such kind of connections enable the lower loading of the network and rapid acquisition of processed information $[3,8]$.

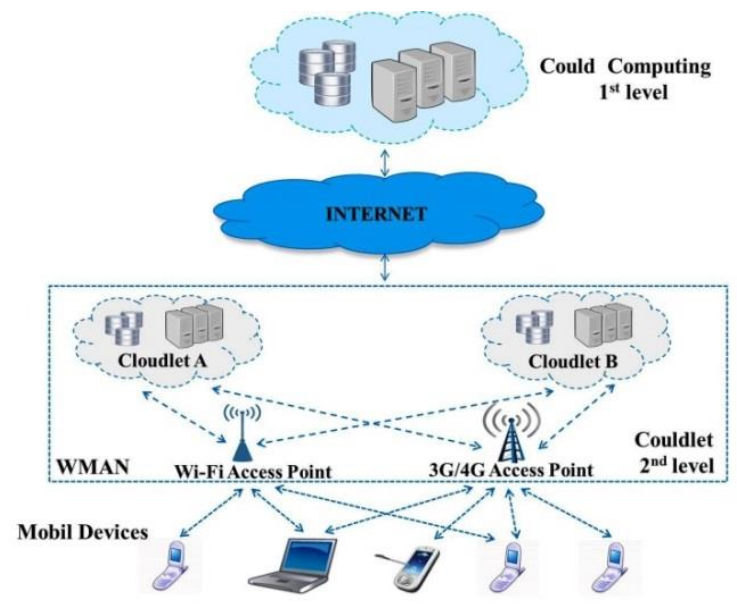

Fig.1. Architecture of Mobile Computing Clouds with Hierarchic Structure

Hence, Cloudlets are created near the base stations of mobile operators for the wider use of cloud technologies by mobile devices. Cloud services required by users are carried out via servers. For instance, should a user need to work with any software tool (SaaS service), he or she uploads the required software to the nearest cloudlet. This, in turn, decreases the price of using the service, reduces the solution time of a problem and the network is not overloaded.

The parameters of server resources (number of virtual machines, memory volume, transmission capabilities of network, etc.) used in developing cloudlets (small clouds) near the base stations is one of the primary issues. Such that, technical facilities of cloudlets must enable the download of software extensions used by users. At the same time, the maintenance of connection among cloudlets is important issue. On the other hand, by using traditional centralized cloud services, the demand for 
high computing and memory resources can be met. However, delays are observed while acquiring the results or data in cloud services. Translation service operating in online regime has been developed by Google (Google Translate API), which can facilitate the communication among users speaking in different languages and communicating via Internet. Mobile devices (smartphones) used in such service do not carry out the translation independently and send the words and sentences to servers of Google Cloud Platform. Translation is carried out in servers and results are delivered to persons communicating with each other. The delays in this type of services are clearly observed. While the translation is done very fast, the delivery of translated material to designated address takes place with delays. Hence, it would be desirable to locate translation software in local servers (cloudlets) close to users and facilitate the translation process in real time regime. At the same time, the location of software tools in cloud servers close to users would lead to cheaper, faster and higher-quality services during the use of SaaS services by users.

While using cloud technologies, users mainly consider the following criteria:

- expenses required for the solution of the problem;

- solution period of the problem;

- provision of the security of user information;

- rapid and secure delivery of data and results to users.

Mentioned criteria hinge on the distance between cloud servers and user, the capacity of virtual computing machine and the loading degree of network. The conduction of services of mobile users in centralized cloud servers is not always economically feasible. Hence, the location of computing and memory resources in cloudlets close to users enables to carry out mentioned service cheaper and with higher quality. For this purpose, it is attempted to locate cloudlets near base stations close to users. This reduces delays and provides the security of users. At the same time, it reduces the demand of mobile device to energy. Thus, cloudlet network infrastructure can be established by connecting cloudlets located close to base stations over the region. Mobile user solves his or her problem by selecting the cloudlet corresponding to established cloudlet network.

\section{RELATED WORK}

The majority of studies conducted in Mobile Cloud Computing technologies is devoted to the issues of efficient organization of the processing of user requests in remotely located cloud servers $[12,13]$. User requests may cause the increase in delays in communication channels (in internet networks, internet providers, among base stations, etc.) due to the overload of internet network in Centralized Cloud Computing systems. In order to eliminate the delays, the establishment of cloudlet networks more closely located users is implemented. In this case, cloudlets process the requests more rapidly and provide less energy consumption of mobile devices as well.

Some research studies explore the conditions necessitating the creation of cloudlets in mobile cloud computing and solve the issue of forecasting of cloudlet location near base stations [14].

The rapid development of cloud computing and the use of various technical and software tools by the growing number of users may cause the disruption of network security and expose the network to various distributed threats. This, in turn, may lead to the violation of the confidentiality in Cloud resources and the emergence of other undesirable events. Hence, some authors propose Collaborative Network Intrusion Detection System (CNIDS) in order to detect the threats to network with the monitoring of network traffic and they utilize Support Vector Machine (SVM) for this purpose [15]. Some authors have developed a powered Cloud IDS prototype via Dendritic Cell mechanism in order to improve security measures [16]. As a result, Dendritic Cell mechanism capable of protecting the human body was successfully implemented in Cloud environment as well.

As a result of studies, it has been revealed that, some users utilizing same virtual machines can engage in harmful activities. Hence, some authors propose elastic and internal protection systems for the purpose of the organization of protection of virtual machines against users [17].

As mentioned above, the number of mobile users utilizing cloud technologies and in particular, number of game players is rapidly growing. It is to be noted that, the interest for intellectual games is related to specific intellectual needs of users and serves to the development of their intellectual capacity [18]. The studies devoted to the development of such games and their closer location to users are being implemented in recent periods [19]. As other software tools used in online regime (translation, navigation, etc.) require an interface with high speed. The solution of this sort of problems necessitates the utilization of cloudlets. The majority of studies conducted in recent periods is oriented towards the solution of the problem of cloudlet use [20].

In some studies [21], it is possible to eliminate the delays by reducing the number of communication channels between users and cloudlets by providing the solution of user requests in a closely located cloudlet. The energy consumption, delays and interruptions can be reduced by correctly locating auxiliary (interface) and main parts of software applications utilized by users in mobile devices and cloud servers.

The lifecycle of cloudlets has been analyzed and some necessary quantitative characteristics have been explored [22]. The solutions has been proposed for the problem of constructing diagrams during the implementation of requests while working with software tools interactively used in On_Line regime. Some authors have considered the issues of services migration in mobile clouds and some advantages of this method has been mentioned $[23,24]$. A simple algorithm can be constructed by 
considering the frequency of requests to software tools in order to implement this process [7]. Each software tool stored in this condition can be deleted from memory resources of a cloudlet when there emerges need for the storage of software tool with higher priority.

\section{PRoBlem StATEMENT}

Cloudlets are developed based on computers (server, work station, desktop computer, netbook, etc.) with various technical features. If computing and memory resources of the Cloudlet located near any network access point are not capable to process the requests of the large number of mobile users, mobile user is forced to use the resources of other Cloudlets located close to him/her. Hence, there emerges a need for establishing the network infrastructure of cloudlets located near various access points.

So, the provision of the connection among Cloudlets is considered to be one of the actual issues. At the same time, Cloudlet resources located close to mobile users may not be vacant resources while being used by other users. In this case, there emerges a need for mobile users to obtain required resources from other Cloudlet resources closely located to those users. On the other hand, often used software tools (depending on the frequency of use) can be stored in Cloudlets for a specific period, so that, other users can use it conveniently.

Conducted monitorings indicate that, the frequency of use of newly launched games by users is usually high. For this purpose, it is feasible to store such games in Cloudlet resources for a specific period. In such case, Cloudlet resources are used for storing this type of program extensions in memory. Mentioned Cloudlet resources are not sufficient for providing services to new mobile users. Thus, the establishment of Cloudlet network infrastructure located close to access points of Wireless Metropolitan Area Networks (WMAN) is one of the important issues in this regard. It is desirable to establish cloudlets near all access points of mentioned network. This is not economically feasible and rises the cost of newly established network. On the other hand, it is possible that, cloudlets located near specific access points are used less or not used at all. Hence, cloudlets are mostly used near the places with many mobile users (shopping malls, libraries, schools, universities, stadiums, stations, airports, etc.) $[9,10]$.

One method of the effective use of cloudlet resources is the download and use of interactive software tools frequently used by users to cloudlet and the storage of those in there as long as the tool is important. It is to be mentioned that, the location of program extensions of mobile users to nearest cloudlet enables the more rapid solution of the problem. Thus, it is attempted to solve the user problem in cloudlets located closer to user in case of absence of important resources in cloudlets to which user is connected. The low number of communication channels between the user and cloudlet can facilitate the realization of operating software tools in shorter period, with higher quality, minimal delays and interruptions
[11]. The proximity is determined by the number of communication channels among cloudlets. On the other hand, program extensions with high frequency of use can be stored in cloudlets for a specific period. So, there is no need for requesting those software extensions from servers of cloud computing system located at long distance $\left(1^{\text {st }}\right.$ level $)$.

Each cloudlet can play a role of a mobile user for some other cloudlets with which it can create a mobile connection. It can implement the solution of specific problems in cloudlets of use or can promptly connect its mobile user with those cloudlets when necessary and possible. This condition facilitates the solution of the problem of loading and storage of On Line software tools in a cloudlet for a specific period. It is possible that, software to be loaded is already loaded to neighboring cloudlets and it can be used or this software tool can be loaded to a cloudlet, which is close for use and used there.

The article presents the analysis of some features of the solution of this problem and the effective use of cloudlets. A solution is proposed to the problem of loading of software tools frequently used by mobile users to one of the cloudlets located on one network and the connection of a user with that cloudlet. For this purpose, the practicable number of routes creating a connection between the user and selected cloudlet on the network is specified. The low number of communication channels between user and a cloudlet leads to more reliable and rapid processing of a problem.

\section{PROPOSED METHOD}

The structural scheme of wireless metropolitan area networks (WMAN) developed on cloudlets is given in figure 2. As seen in the scheme, cloudlets are located in the proximity of important access points according to the place.

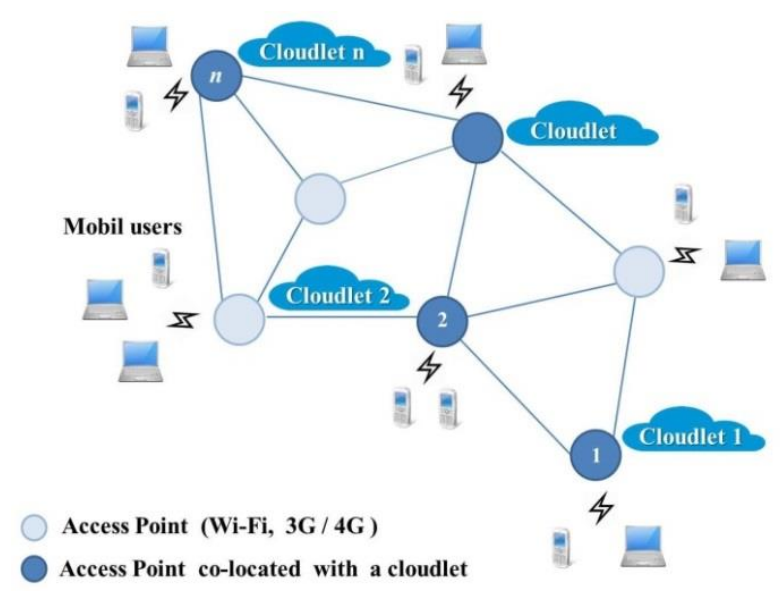

Fig.2. Network Infrastructure based on Cloudlet

Denote the network infrastructure, the cloudlet storing Online software tool or software tools and other cloudlets that the cloudlet can easily connect with mobile tools as network of cloudlets. It is assumed that, each of the cloudlets on the network is capable to communicating 
with each other. Let's assume that, $\mathrm{N}$ is the number of cloudlets in the network. Each cloudlet can directly communicate with $\mathrm{N}-1$ number of other cloudlets.

Denote the software extension required for use by mobile users as $P A_{s}$. Assume that, a mobile user connected to $i^{\text {th }}$ cloudlet will use this software tool. Denote existing vacant memory space for storing new tools in ith cloudlet as $V_{e}(i)$ and the memory space required for a software tool $\left(\mathrm{PA}_{\mathrm{s}}\right)$ to be used as $V\left(P A_{s}\right)$. It is clear that, in a simple case, if

$$
V\left(P A_{s}\right)<V_{e}(i)
$$

is satisfied, a new software tool can be stored in $i^{\text {th }}$ cloudlet. If condition (1) is not met, $P A_{s}$ software tool required to be stored can be stored in one of the cloudlets located at the same network as ith Cloudlet.

While locating Online software tool in other cloudlets and if this tool is located in other cloudlet of the same network, a mobile user can be connected to that cloudlet. For this purpose, a set of software tools stored in cloudlets in the network must be analyzed. Assume that, a set of software tools stored in $i^{\text {th }}$ cloudlet is $P A(i)=$ $\left\{P A_{i 1}, P A_{i 2}, \ldots, P A_{i, i_{k}}\right\}$ set. This set can be corrected as software tools that are added or removed and transmitted to other cloudlets at the same network. Hence, a set of software tools located at the same network can be expressed as

$$
P A=\bigcup_{i=1}^{N} P A(i)
$$

If a cloudlet is found to satisfy

$$
P A_{s} \in P A=\bigcup_{i=1}^{N} P A(i)
$$

then, a mobile user intending to use with $P A_{s}$ software tool can be connected with those cloudlets. A problem can have such a technical solution that, a mobile user would not notice the realization of this procedure.

If (3) condition is not satisfied, the following algorithms can be applied.

It is clear that, the total number of connections linking to different cloudlets at the network can be defined at maximum as below:

$$
L=(N-1)+(N-2)+\cdots+2+1=\frac{N(N-1)}{2}
$$

In general, at each of these connections:

- Transmission speed $v_{l} ; l \in[1, L]$;

- Transmission probability $p_{l} ; l \in[1, L]$; etc. can be determined.

It is to be noted that, these parameters are changeable in the course of time. It is caused by factors such as the change in technical equipment, depreciation of some equipment in time and the change of characteristics. However, we can take these parameters as constant within some practical time period.

Let's consider the number of routes connecting one cloudlet ( $i^{\text {th }}$ cloudlet) with second designated cloudlet $\left(j^{\text {th }}\right.$ cloudlet). One connection links $i^{\text {th }}$ and $j^{\text {th }}$ cloudlets. Routes created between two designated cloudlets can pass through some or all of $\mathrm{N}-2$ number of other cloudlets different from designated cloudlets. The number of created routes by passing through these intermediate cloudlets is

$$
A_{N-2}^{1}=\frac{(N-2) !}{(N-2-1) !}=N-2,
$$

The number of routes to be created by passing through to intermediate cloudlets is

$$
A_{N-2}^{2}=\frac{(N-2) !}{(N-2-2) !}=(N-2)(N-3) \text {. }
$$

In general, the total number of routes linking two cloudlets can be

$$
M=\sum_{k=0}^{N-2} A_{N-2}^{k}
$$

Here,

$$
A_{N-2}^{k}=\frac{(N-2) !}{(N-2-k) !} \text { and it shows the number of various }
$$
sets that can be created with $\mathrm{k}$ element in each out of $\mathrm{N}-2$ elements [25,26].

While the number of routes calculated with the formula (5) is high, it may be too complicated for use for practical purposes. Hence, the practical number of routes connecting two cloudlets can be calculated directly and by taking the number of intermediate cloudlets maximum as 2 as below for $N \geq 3$ :

$$
\begin{gathered}
M=A_{N-2}^{0}+A_{N-2}^{1}+A_{N-2}^{2}=\frac{(N-2) !}{(N-2) !}+\frac{(N-2) !}{(N-3) !}+\frac{(N-2) !}{(N-4) !} \\
M=N^{2}-4 \mathrm{~N}+5
\end{gathered}
$$

While the length of routes is corresponding to the number of connections included in routes, the length can be taken as equal to the number of connections for simplicity. Given that, we have taken the maximum number of intermediate cloudlets as 2 in formula (6), the minimum and maximum number of connections in routes linking two cloudlets can be 1 and 3, respectively.

Here, we assume that, if the number of intermediate cloudlets is less than 3 , several routes emerge connecting two cloudlets and one of them will be selected for problem solution. Surely, theoretically, the routes passing through intermediate cloudlets can also be used when necessary. In this case, the number of additional connection channels to be created can be calculated according to (5) and (6) formulas analogically. However, application of intermediate cloudlets in most cases can reduce the reliability of such connection channels. Hence, the maximum number of intermediate cloudlets can be 2 for practical purposes. That is:

let's denote the number of connection channels at $m^{\text {th }}(m \in[1, M])$ route as $r_{m}$. In this case, $1 \leq r_{m} \leq 3$. Routes connecting $i^{\text {th }}$ cloudlet to $j^{\text {th }}$ cloudlet can be 
denoted as $m^{i j}\left(m^{i j} \in[1, M]\right)$ and the number of connections in these routes as $r_{m}^{i j}$. The probability of transmission of connections included in each of these routes are selected from $p_{l}(l \in[1, L])$ and can be specified as $p_{m}^{i j}(k),\left(k=1, \ldots r_{m}^{i j}\right)$. While calculating the probability of connection linking $i^{\text {th }}$ and $j^{\text {th }}$ cloudlet, the following must be considered:

- Each route of connection $i j$ is the sequential link of communications. Hence, transmission probability of $m^{i j}\left(m^{i j} \in[1, M]\right)$ route can be calculated as

$$
p_{m}^{i j}=\prod_{k=1}^{r_{m}^{i j}} p_{m}^{i j}(k)
$$

- It is assumed that, routes in connection $i j$ connect $i^{\text {th }}$ cloudlet and $j^{\text {th }}$ cloudlet in parallel. So, the final probability of transmission is:

$$
p^{i j}=1-\prod_{m=1}^{M}\left(1-p_{m}^{i j}\right) \text { olar }, i \neq j
$$

While solving the issue of locating software tool to any cloudlet, potential cloudlets must be evaluated by $i^{\text {th }}$ cloudlet. During evaluation, quantitative and qualitative features of a cloudlet must be taken into consideration. For this purpose, fuzzy and other methods can be applied [27-29]. We do not consider the evaluation problem mentioned above. However, it is clear that, such evaluation can be conducted intuitively. Hence, this value can be considered as integral value of a cloudlet and denoted as $I C(j)$ for each cloudlet.

While determining the significance of any cloudlet on this network for problem solution, $I C(j)$ must be multiplied to $p^{i j}$ probability of transmission of $i j$ connection. This product will consider the significance of cloudlet, as well as the quality of connection. A cloudlet with large $p^{i j} * I C(j)$ product and satisfying condition (1) can be selected.

So, $P A_{s}$ software tool can be located in $j^{\text {th }}$ cloudlet colocated with $i^{\text {th }}$ cloudlet on the same network and satisfying the following conditions:

$$
\left\{\begin{array}{c}
p^{i j} * I C(j)=\max \left\{p^{i z} * I C(z)\right\}, z \neq i, j \neq i \\
V\left(P A_{s}\right)<V_{e}(z), z \neq i
\end{array}\right.
$$

So, the requested software tool can be uploaded to $j^{\text {th }}$ cloudlet satisfying condition (8) and mobile users requiring to work with this software tools can be connected to that cloudlet. Thereafter, a set of software tools of $j^{\text {th }}$ cloudlet can be corrected and sent to other cloudlets. As a result, cloudlets located at one place can process the requests of mobile users more effectively.

\section{CONCLUSION}

The article presents a method for maintaining the effective realization of requests of mobile users utilizing the cloudlets. In this case, the cloudlets in networks are defined and the set of such cloudlets are specified. A solution is proposed for the problem of uploading online software tools frequently used by mobile users to one of the cloudlets located on the network and the connection of mobile user with specific cloudlet. For this purpose, the architecture of mobile cloud technologies with hierarchical structure has been explored and main problematic issues of mobile cloud computing has been analyzed. The methods of eliminating some delays emerging in network during the use of Mobile Cloud Computing technologies has been proposed. The practical number of routes creating a connection between two cloudlets located on one plane has been specified in order to provide the efficient use of cloud technologies and cloudlet resources by mobile users. The probability of routes and the whole connection is determined by using transmission probabilities between two cloudlets. The conditions for uploading necessary software tools to a specific cloudlet is determined by using these probabilities and possible values specifying the importance of cloudlets.

The method and obtained results in the article can be used for the solution of similar problems in local networks.

\section{ACKNOWLEDGMENT}

This work was supported by the Science Development Foundation under the President of the Republic of Azerbaijan - Grant № EİF-2014-9(24)-KETPL-14/02/1.

\section{REFERENCES}

[1] Alguliyev R.M., Alekperov R.K. Cloud Computing: Modern State, Problems and Prospects// Telecommunications and Radio Engineering, 2013, vol.72, no.3, pp. 255-266.

[2] Sumit Goyal. Public vs Private vs Hybrid vs CommunityCloud Computing: A Critical Review. I.J. Computer Network and Information Security, 2014, 3, pp. 20-29. Published Online February 2014 in MECS (http://www.mecs-press.org/) DOI: 10.5815/ijcnis.2014.03.03

[3] Hoang T. Dinh, Chonho Lee, Dusit Niyato, and Ping Wang. A survey of mobile cloud computing: Architecture, applications, and approaches. Wireless Communications and Mobile Computing. Volume 13, Issue 18. 25 December 2013, pp. 1587-1611.

[4] Han Qi, Abdullah Gani. Research on Mobile Cloud Computing: Review, Trend and Perspectives. https://arxiv.org/ftp/arxiv/papers/ 1206/1206.1118.pdf

[5] Mukesh Goyal, Sukhwinder Singh. Mobile Cloud Computing. International Journal of Enhanced Research in Science Technology \& Engineering, ISSN: 2319-7463 vol.3, Issue 4, April-2014, pp. 517-521.

[6] Alakbarov R.G., Alakbarov O.R. Mobile Clouds Computing: Current State, Architecture and Problems. 2nd IEEE International Conference on Electrical, Computer and Communication Technologies (IEEE ICECCT 2017) 22 - 24, February 2017, Coimbatore, India. 10.1109/ICECCT.2017.8117874

[7] Fernando N., Seng L.W., Rahayu L. Mobile Cloud Computing: A Survey // Journal of Future Generation System, 2013, vol29, no.1, pp. 84-106.

[8] Fangming Liu, Peng Shu, Hai Jin, Linjie Ding, Jie Yu, Di Niu, Bo Li. Gearing resource-poor mobile devices 
with powerful clouds: architectures, challenges, and applications. IEEE Wireless Communications. vol.20, Issue: 3, June 2013, pp.14-22.

[9] M. Jia, J. Cao, and W. Liang. Optimal cloudlet placement and user to cloudlet allocation in wireless metropolitan area networks. To appear in IEEE Transactions on Cloud Computing.Vol.PP.Issue:3.June2015.http://users.cecs.anu .edu.au/ Weifa.Liang/publication.html.

[10] Alakbarov R.G., Pashayev F.H., Alakbarov O.R. Optimal Deployment Model of Cloudlets In Mobile Cloud Computing. 2nd IEEE International Conference on Cloud Computing and Big Data Analysis (IEEE ICCCBDA 2017). April 28-30, 2017, Chengdu, China. pp. 213-217

[11] Zichuan Xu, Weifa Liang, Wenzheng Xu, Mike Jia, and Song Guo. Efficient Algorithms for Capacitated Cloudlet.Placements. IEEE Transactions on Parallel and Distributed Systems, Volume: 27, Issue: 10, Oct. 1 2016 .Page(s):2866- 2880.

[12] Young-Chul Shim. Effects of cloudlets on interactive applications in mobile cloud computing environments. International Journal of Advanced Computer Technology, Volume 4, Number 1, 2015, pp.54-62.

[13] Oladeji P. Akomolafe. Matthew O. Abodunrin. A Hybrid Cryptographic Model for Data Storage in Mobile Cloud Computing.I. J. Computer Network and Information Security, 2017, 6, pp.53-60. Published Online June 2017 in MECS (http://www.mecs-press.org/) DOI: 0.5815/ijcnis. 2017.06.06

[14] Alakbarov R., Pashayev F., Alakbarov O. Forecasting Cloudlet Development on Mobile Computing Clouds // I.J. Information Technology and Computer Science, 2017, Vol.9, No.11, pp.23-34.

[15] Zayed Al Haddad, Mostafa Hanoune and Abdelaziz Mamouni. A Collaborative Network Intrusion Detection System (C-NIDS) in Cloud Computing. International Journal of Communication Networks and Information Security (IJCNIS) Vol. 8, No. 3, December 2016, pp. 130-135.

[16] Azuan Ahmad, Norbik Bashah Idris and Mohd Nazri Kama. CloudIDS: Cloud Intrusion Detection Model Inspired by Dendritic Cell Mechanism. International Journal of Communication Networks and Information Security (IJCNIS) Vol. 9, No. 1, April 2017. Pp. 67-75.

[17] J. D. Araújo, D. de Andrade Rodrigues, L. S. de Melo, and Z. Abdelouahab, "EICIDS-elastic and internal cloudbased detection system, International Journal of Communication Networks and Information Security, vol. 7, p. 34, 2015. pp. 34-49.

[18] Ryzhov A.L., Tkhostov A., Pechnikova L. Psychological aspects of involvement in Massively Multiplayer On-line Game (MMOG). International Journal of Psychology. 2015. том 51, № S1, pp.594.

[19] Eric Lengyel. Foundations of Game Engine Development. Terathon Software LLC. 2016. 200 p.

[20] Joshua Glazer, Sanjoy Madhav. Multiplayer Game Programming. Architecting Networked Games. Pearson Edication Inc. 2015. 365 p.

[21] Usman Shaukat, Ejaz Ahmed, Zahid Anwar, Feng Xia. Cloudlet deployment in local wireless networks: Motivation, architectures, applications, and open challenges Journal of Network and Computer Applications 62 (2016) pp. 18-40

[22] Debessay Fesehaye, Yunlong Gao, Klara Nahrstedt, Guijun Wang. Impact of Cloudlets on Interactive Mobile Cloud Applications. Enterprise Distributed Object Computing Conference (EDOC), 2012 IEEE 16th International, pp.10.

[23] Yujin Li and Wenye Wang. Can Mobile Cloudlets Support Mobile Applications? https://research.ece.ncsu.edu/netwis/papers /14LWInfo.pdf

[24] S. Wang, R. Urgaonkar, M. Zafer, T. He, K. Chan, and K. $\mathrm{K}$. Leung, "Dynamic service migration in mobile edgeclouds," in Proc. of IFIP Networking 2015, May 2015, pp 9.

[25] Eddous M., Stansfield R. Methods of decision-making. Moscow: Audit, UNITI, 1997. 590 p.

[26] T.Stewart. Calculus: Early Transcendentals, Seventh Edition, Brooks/Cole, Cengage Learning, 2012, 1356 p.

[27] R. E. Bellman, L. A. Zadeh, Decision-Making in a Fuzzy Environment, Management Science, 17, № 4, 1970. pp. $141-164$.

[28] T.L. Saati. Decision making with the analytical hierarchical process. M: Radio and communication. 1993. $278 \mathrm{p}$.

[29] L. A. Zadeh. Definition of linguistic variable and its implementation to approximate decision-making. M. Mir, 1976. $165 \mathrm{p}$.

\section{Authors' Profiles}

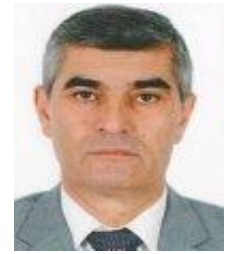

Rashid G. Alakbarov graduated fromAutomation and Computer Engineeringl faculty of Azerbaijan Polytechnic University named after C.Ildirim. He received his $\mathrm{PhD}$ degree in 2006 from Supreme Attestation Commission under the President of the Republic of Azerbaijan. His primary research interests include various areas in cloud computing, data processing, computer networks, virtual computing, particularly in the area of distributed computing. He is head of department at the Institute of Information Technology as of 2002. Since 2010, he has been leading the development of "AzScienceNet" infrastructure. In 2011, he was appointed a deputy director of the institute by the decision of the Presidium of Azerbaijan National Academy of Sciences. He is the author of 70 scientific papers, including 5 inventions.

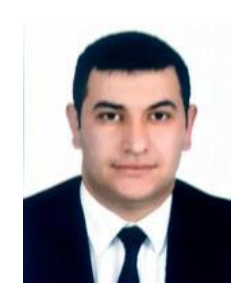

Oqtay R. Alakbarov is $\mathrm{PhD}$ student of Institute of Information Technology of Azerbaijan National Academy of Sciences. His primary research interests include various areas in cloud computing, mobile cloud computing, mobile technologies, particularly in the area of cloud technology applications. He is the author of 3 journal scientific papers and 3 proceedings.

How to cite this paper: Rashid G. Alakbarov, Oqtay R. Alakbarov,"Effective use Method of Cloudlet Resources by Mobile Users", International Journal of Computer Network and Information Security(IJCNIS), Vol.10, No.2, pp.46-52, 2018.DOI: 10.5815/ijcnis.2018.02.06 